\title{
UTILIZAÇÃO DO PACLOBUTRAZOL, ETHEPHON E NITRATO DE POTÁSSIO NA INDUÇÃO FLORAL DA MANGUEIRA NO SEMI-ÁRIDO NORDESTINO ${ }^{1}$
}

\author{
VANDER MENDONÇA ${ }^{2}$ \\ JOSÉ DARLAN RAMOS ${ }^{3}$ \\ JOSIVAN BARBOSA MENEZES ${ }^{4}$ \\ RENATO INNECCO ${ }^{5}$ \\ RAFAEL PIO ${ }^{6}$
}

\begin{abstract}
RESUMO - Com este trabalho objetivou-se verificar o comportamento produtivo da cultura da manga, cultivar Tommy Atkins, com uso do paclobutrazol, nitrato de potássio e ethephon, conduzida em plantios comerciais da MAÍSA (Mossoró Agroindústrial Sociedade Anônima). O experimento foi desenvolvido no ano de 1999 em delineamento experimental de blocos casualizados, no esquema fatorial $3 \times 2 \times 3 ; 3$ doses de Paclobutrazol: 0 mg.L $\mathrm{L}^{-1} 1000 \mathrm{mg} . \mathrm{L}^{-1}$ e $1500 \mathrm{mg} . \mathrm{L}^{-1} ; 2$ concentrações de
\end{abstract}

nitrato de potássio: 3 e 4\%; 3 dosagens de ethephon: 0 mL. $\mathrm{L}^{-1}, 0,50 \mathrm{~mL} . \mathrm{L}^{-1}$ e $1,0 \mathrm{~mL} . \mathrm{L}^{-1}$, com 4 repetições. $\mathrm{O}$ paclobutrazol na dosagem de $1500 \mathrm{mg} . \mathrm{L}^{-1}$ foi a que mais reduziu o crescimento das plantas. Esse produto apresentou-se como um bom inibidor de crescimento e teve boas respostas no rendimento da mangueira cultivar Tommy Atkins. $\mathrm{O} \mathrm{KNO}_{3}$ e o ethephon, isoladamente, não influenciaram em nenhuma das características avaliadas.

TERMOS PARA INDEXAÇÃO: Mangifera indica L., indução floral, produção.

\section{UTILIZATION OF PACLOBUTRAZOL, ETHEPHON AND POTASSIUM NITRATE IN THE FLOWERING INDUCTION OF MANGO IN THE SEMI ARID NORTHEST}

\begin{abstract}
An experiment was conducted in order to verify the effect of different concentrations of Paclobutrazol, Ethephon and Potassium Nitrate in the production of mango cv. Tommy Atkins at agricultural area of Mossoró-Assu. The experiment was carried out in 1999, under an experimental design of randomized blocks, with an factorial arrangement of $3 \times 2 \times 3$ (dose of Paclobutrazol: 0 mg.L $\mathrm{L}^{-1}, 1000 \mathrm{mg} . \mathrm{L}^{-1}$ and $1500 \mathrm{mg} . \mathrm{L}^{-}$
\end{abstract}

\begin{abstract}
${ }^{1}$ ); 2 (Potassium Nitrate concentrations: 3 and 4\%); 3 (dose of Ethephon: 0,0 mL.L ${ }^{-1}, 0,50 \mathrm{~mL} . \mathrm{L}^{-}$and 1,0 mL.L ${ }^{-}$) with four replicates. The dosage of 1500 mg. $\mathrm{L}^{-1}$ of Paclobutrazol caused the largest reduction of plants growth. This product showed good growth inhibition characteristics. The $\mathrm{KNO}_{3}$ and Ethephon, separately, did not influence none of the evaluated characteristics.
\end{abstract}

INDEX TERMS: Mangifera indica L., floral induction, production.

1. Trabalho extraído da dissertação do primeiro autor, apresentada à Escola Superior de Agricultura de Mossoró/ ESAM-RN.

2. Engenheiro Agrônomo, M.Sc. Doutorando, UNIVERSIDADE FEDERAL DE LAVRAS/UFLA - Caixa Postal 37 37200-000 - Lavras, MG, vander@ufla.br

3. Engenheiro Agrônomo, Professor, Dr. Departamento de Agricultura/UFLA, darlan@ufla.br

4. Engenheiro Agrônomo, Professor, Dr. Departamento de Química da ESAM, 59625-900 - Mossoró-RN.

5. Engenheiro Agrônomo, Professor, Dr. Universidade Federal do Ceará/UFC - Fortaleza, CE.

6. Engenheiro Agrônomo, M.Sc. Doutorando-Agronomia/Fitotecnia - ESALQ/USP - Piracicaba, SP. rafaelpio @hotmail.com 


\section{INTRODUÇÃO}

No Rio Grande do Norte, o cultivo da manga tipo exportação encontra-se em fase de grande expansão, tendo como base as cultivares Tommy Atkins, Van Dyke e Haden. Os principais plantios estão localizados no município de Mossoró e no Vale do Assu.

Os principais problemas enfrentados pelos produtores de manga no Brasil e no mundo são o baixo rendimento da planta e a floração com elevados índices de queda de frutos durante seu desenvolvimento, em que menos de $1 \%$ dos frutos atinge o estádio de maturação (SIMÃO, 1971). Assim, muitos produtores, principalmente na Região Nordeste, estão utilizando produtos como nitratos e ethephon juntamente com estresse hídrico, no intuito de provocar um maior florescimento e rendimento da planta, embora esses produtos ainda necessitem de mais estudos que demostrem sua eficiência na indução floral e no rendimento da planta.

Em trabalhos com indução, Nuñez-Elizea e Caldeira (1987), Albuquerque e Medina (1991), Barros et al. (1996) e Santana et al. (1997), conseguiram com sucesso induzir o florescimento da mangueira com aplicações de $\mathrm{KNO}_{3}$ em concentrações que variam de 2 a 6\%. Porém, alguns pesquisadores como Pal et al. (1984) e Valente e Donadio (1993) não obtiveram sucesso nessa mesma prática.

Alguns fatores, como concentração de produto na calda, horário e freqüência das aplicações, falta de estresse hídrico adequado sofrido pelas plantas e até mesmo o estado nutricional e fisiológico das plantas na época da indução (WINSTON e WRIGHT, 1984; ALBUQUERQUE e MEDINA, 1991; TANAJURA FILHO, 1992; SILVA et al. 1994; CASTRO NETO, 1995) podem estar associados aos resultados desfavoráveis encontrados na indução de florescimento pela aplicação de $\mathrm{KNO}_{3}$ e ethephon.

Uma outra prática que está sendo utilizada, aliada à aplicação de indutores de florescimento, é a aplicação de produtos que restringem o crescimento vegetativo das plantas, como o paclobutrazol. Várias cultivares de manga têm sido tratadas com esse regulador, conforme Winston (1992), Kurian e Iyer (1993), Ferrari e Sergent (1996a) e Salazar-Garcia e Vazquez-Valdina (1997) e, em geral, os efeitos parecem comuns, contudo, pouco se conhece sobre os efeitos desse produto nos processos fisiológicos das plantas (WAMPLE e CULVER, 1983).

Objetivou-se com este trabalho verificar o comportamento da mangueira cultivar Tommy Atkins, submetida a aplicações de Paclobutrazol (PBZ), ethe- phon e nitrato de potássio, na região semi-árida do nordeste brasileiro.

\section{MATERIAL E MÉTODOS}

Este trabalho foi conduzido nos meses de maio dezembro de 1999/2000, em plantio comercial de mangueira cv.Tommy Atkins localizado na fazenda Jaísa, na cidade de Jaguaruana-CE, de propriedade da MAÍSA (Mossoró Agroindústrial Sociedade Anônima) situada no Pólo Agrícola Mossoró-Assu-RN.

O pomar tinha cinco anos e foi instalado sobre o porta enxerto Espada com espaçamento de $10 \mathrm{~m} \mathrm{x}$ $10 \mathrm{~m}$. O solo da região é do tipo arenoso e as plantas eram irrigadas com sistema de irrigação por microaspersão, com gasto de $140 \mathrm{~L}$ de água/dia planta no pico de irrigação.

Os tratos culturais foram os normalmente recomendados para a cultura (CUNHA et al. 1994). Foi feita também, logo após a última colheita, uma poda de abertura de copa nas plantas antes da aplicação do paclobutrazol.

$\mathrm{O}$ delineamento experimental utilizado foi em blocos casualizados no esquema fatorial $(3 \times 2 \times 3)$, com 18 tratamentos e quatro repetições, sendo três dosagens de paclobutrazol (PBZ a 10\%) (0; 1000 mg.L $\mathrm{L}^{-1}$ e 1500 mg. $\left.\mathrm{L}^{-1}\right), 2$ concentrações de nitrato de potássio ( 3 e $4 \%$ ) e 3 dosagens de ethephon ( 0,$0 ; 0,50$ mL. $\mathrm{L}^{-1}$ e 1,0 $\mathrm{mL} . \mathrm{L}^{-1}$ ). Para a dosagem 0 (zero), tanto do paclobutrazol, como do ethephon, usou-se água pura. Nas aplicações do ethephon e do nitrato, foi utilizado espalhante adesivo (alquil fenol poliglicoléter $250 \mathrm{~g} . \mathrm{L}^{-1}$ ) de nome comercial Adesil, na dosagem de 0,4 mL. $\mathrm{L}^{-1}$ de calda. As aplicações foram feitas à tarde, por volta de 17 horas, sendo utilizados em média dez litros da solução por planta, volume suficiente para garantir uma cobertura completa da copa até o ponto do início do escorrimento foliar da calda.

Foi utilizada uma planta por parcela e, para as análises das características, foram marcados 25 galhos por parcela com média de 80 ramos terminais. Desses ramos terminais, posteriormente, selecionaram-se seis ao acaso por planta, provenientes de galhos situados nos quatros quadrantes da planta a uma altura em torno de 1,5 a 2,5 m. Nesses seis ramos terminais observouse quais apresentaram panícula.

O paclobutrazol foi aplicado uma única vez, via solo, no dia 25/05/99, em sulcos feitos com as dimensões de $1 \mathrm{~m}$ de comprimento, $20 \mathrm{~cm}$ de largura e $10 \mathrm{~cm}$ de profundidade, a uma distância de $30 \mathrm{~cm}$ do colo da planta. Nesses sulcos foi adicionado o produto dissolvi- 
do em 2 litros de água nas concentrações indicadas nos tratamentos.

Em torno de sessenta dias após a aplicação do paclobutrazol, iniciaram-se as aplicações do ethephon, que foram feitas duas vezes, via foliar, sendo a primeira no dia 26/07/99 e a segunda no dia 09/08/99. As aplicações foram feitas dissolvendo-se o produto nas concentrações, conforme os tratamentos, em um pulverizador com de 400 litros de água. $\mathrm{O} \mathrm{KNO}_{3}$ foi aplicado cinco vezes, semanalmente, com início em 23/08/99, 14 dias após a última aplicação do ethephon e término em 20/09/99, utilizando-se mesma metodologia de aplicação usada nas aplicações do ethephon. Logo após a primeira aplicação do ethephon (26/07/99), foi reduzida a lâmina d'água para $14 \mathrm{~L} /$ dia/planta, mantida até a segunda aplicação do $\mathrm{KNO}_{3}(30 / 08 / 99)$.

Características estudadas: Crescimento de plantas (CP): foram feitas duas medidas na altura das plantas; uma no dia da aplicação do paclobutrazol (25/05/1999) e a outra após 86 dias da aplicação do PBZ (19/08/1999); em seguida, subtraiu-se a última leitura com a primeira, encontrando, assim, o valor do crescimento das plantas no período de atuação do produto. Para essas leituras, foi utilizado uma régua graduada, medindo-se do colo até o ápice da copa da planta. Tempo para atingir $50 \%$ do florescimento $(\mathrm{T} 50 \% \mathrm{~F})$ : após a terceira aplicação de $\mathrm{KNO}_{3}$, quando já se observava o início do florescimento (Estádio $\mathrm{C}$ ), segundo Albert e Lossois (1972), iniciou-se a contagem do tempo para que se atingisse $50 \%$ do florescimento. Para observar o florescimento, visitava-se semanalmente o pomar para verificar se os ramos previamente marcados estavam floridos. Taxa de florescimento (TF): após a completa floração, observou-se nos ramos terminais, previamente marcados (80 em média por parcela), quais tinham flores. Número e peso médio de frutos por planta: após a colheita, os frutos foram separados por tratamento, pesados e contados e, em seguida, dividiu-se o número total de frutos pelo peso total, obtendose, assim, o peso médio de cada fruto nos diferentes tratamentos; avaliou-se também a produção total de frutos por planta e por hectare.

As análises de variância foram realizadas pelo aplicativo do "software" SPSSPC, (NORUSIS, 1990), e as regressões, pelo software Excel.

\section{RESULTADOS E DISCUSSÃO}

O tratamento de maior dosagem de paclobutrazol (1500 mg...-1) foi o que proporcionou um menor crescimento das plantas $(7,75 \mathrm{~cm})$ Figura 1. Após 86 dias da aplicação do paclobutrazol, pode-se observar no campo um escurecimento das folhas, bem como a paralisação do crescimento vegetativo. Esses sintomas são atribuídos ao princípio ativo do PBZ que, uma vez absorvido pelas raízes, segundo Wiston (1992), vai atuar diretamente na inibição da biossíntese das giberelinas. Com isso, a planta pára de emitir novos ramos vegetativos, paralisando o seu crescimento e os ramos já emitidos amadurecem tornando-se aptos para a indução floral.

Kurian e Iyer (1993) obteve semelhantes resposta em relação à diferença de altura de plantas de mangueira. Salazar-Garcia e Vazquez-Valdina (1997), aplicando diferentes concentrações desse mesmo produto em mangueira cultivar Tommy Atkins, obtiveram melhores respostas em termos de redução de altura de plantas na concentração de $10 \mathrm{~g}(25 \mathrm{ml})$ por planta.

A resposta para a característica tempo para atingir 50\% do florescimento foi significativa apenas nos tratamentos com o paclobutrazol. Analisando-se a Figura 1, observa-se que as dosagens do PBZ tiveram influência distintas na antecipação do florescimento. No tratamento com dosagem zero de paclobutrazol, ou seja, sem a aplicação, o tempo gasto para atingir $50 \%$ do florescimento ficou em torno de 27 dias, já na dosagem $1000 \mathrm{mg} . \mathrm{L}^{-1}$ de paclobutrazol esse tempo foi reduzido para 12 dias e, na dosagem de $1500 \mathrm{mg} . \mathrm{L}^{-1}$, o tempo voltou a aumentar (20 dias).

Essas observações vêm confirmar os resultados obtidos em vários trabalhos na antecipação do florescimento em mangueira com utilização do paclobutrazol (FERRARI e SERGENT, 1996b; SALAZAR-GARCIA e VAZQUEZ-VALDINA, 1997; ALBUQUERQUE et al., 1999).

Neste trabalho não foi observado o efeito significativo das duas concentrações de nitrato de potássio (3 e 4\%) em nenhuma das características estudadas. Possivelmente o elevado teor de umidade no solo em função de irrigações constantes na época de aplicação do $\mathrm{KNO}_{3}$, interagindo com o nitrogênio desse produto, tenha prejudicado seu efeito, uma vez que, para que esse produto tenha um melhor efeito, é necessário que as plantas estejam sob efeito de estresse hídrico, o que não foi possível. Rabêlo (1995), trabalhando com a cultura da mangueira 'Haden' utilizando o anelamento com aplicações de ethephon e $\mathrm{KNO}_{3}$, observou também que o tratamento que só recebeu $\mathrm{KNO}_{3}$ não teve efeito na antecipação do florescimento. 
O efeito do ethephon também não foi significativo na antecipação do florescimento, gastando, em média, 22, 21 e 20 dias para atingir metade do florescimento nas doses 0,$0 ; 0,50 \mathrm{~mL} . \mathrm{L}^{-1}$ e $1,0 \mathrm{~mL}$. $\mathrm{L}^{-1}$, respectivamente. Talvez o estado fisiológico, nutricional, idade da planta e até mesmo temperatura e umidade (Figura 2) possam ter influenciado no efeito do ethe- phon no florescimento, segundo relata Castro Neto (1995). A queda na umidade relativa do ar (Figura 2), observada durante a condução deste trabalho, pode ter causado um aumento no déficit de vapor, induzindo, assim, uma situação de estresse hídrico na planta, mascarando a ação do ethephon. Reis (1999) verificou também que variações na temperatura inibiu a ação do ethephon.

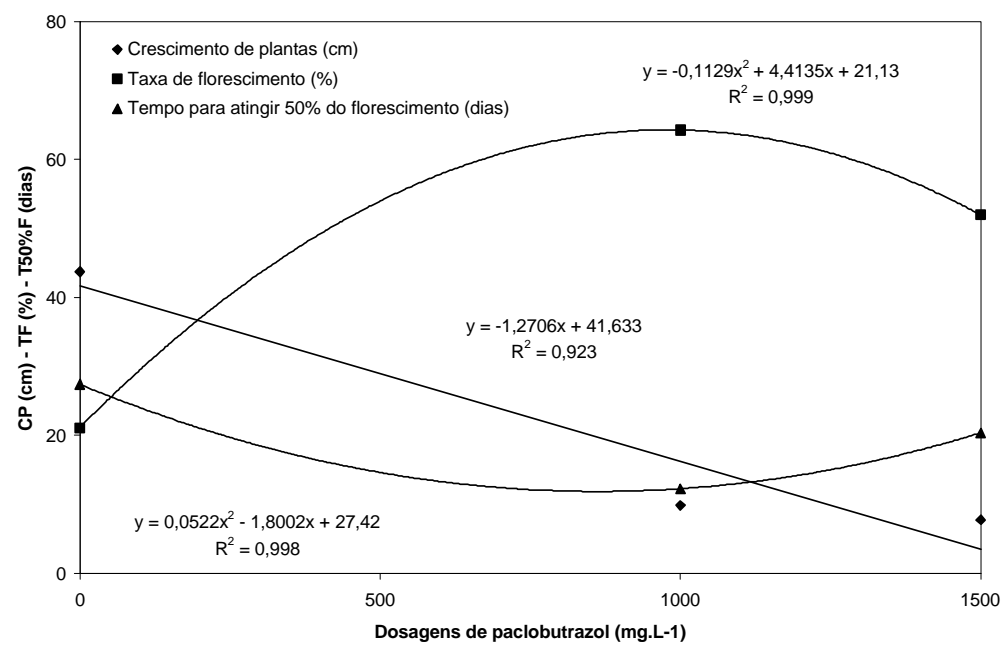

FIGURA 1 - Equação de regressão para a característica crescimento de plantas (CP) de mangueira após 86 dias da aplicação de PBZ); taxa de florescimento (TF) e tempo para atingir 50\% do florescimento (T50\%F). ESAM, Mossoró - RN, 2000.

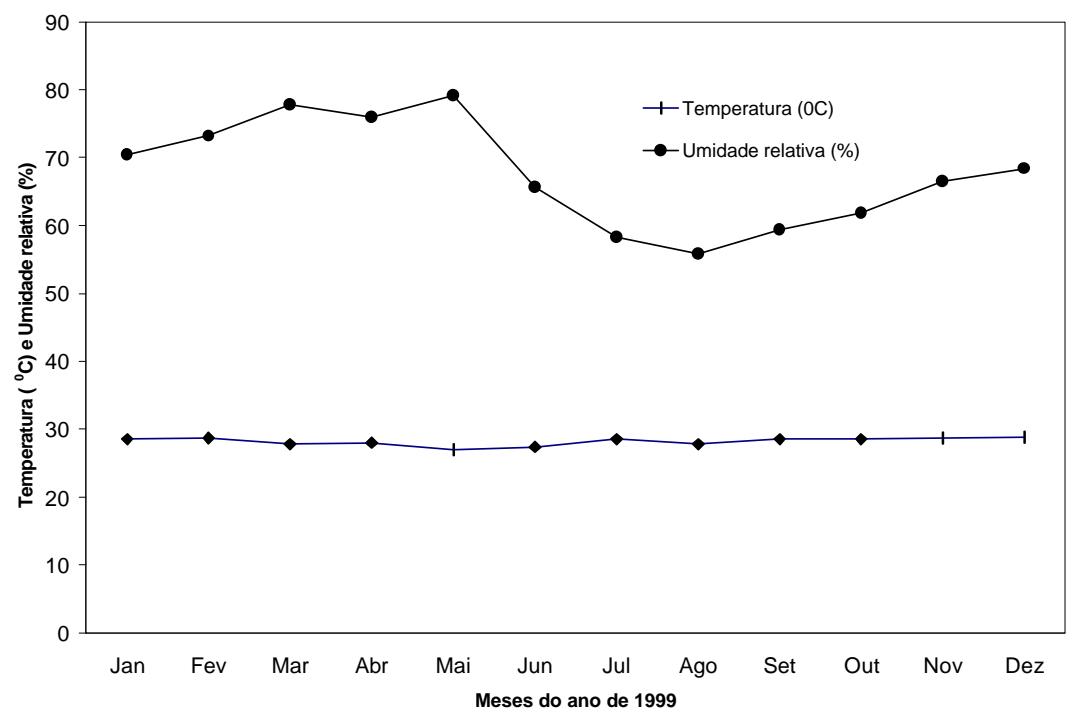

FIGURA 2 - Temperatura média $\left({ }^{\circ} \mathrm{C}\right)$ e Umidade Relativa $(\%)$ na região próxima ao pomar onde foi conduzido o experimento. ESAM, Mossoró - RN, 2000. 
O florescimento representa o número de galhos terminais previamente marcados (80 por parcela) que floresceram. Constatou-se que houve diferenças significativas apenas para o tratamento com paclobutrazol.

À medida que se aumenta a dosagem do (PBZ), a taxa de florescimento também aumenta, e a dosagem de 0 (zero) a $1000 \mathrm{mg} . \mathrm{L}^{-1}$ de paclobutrazol houve um aumento de $64,3 \%$ no florescimento e este aumento é reduzido para 51,9\% na dosagem de 1500 mg.L - $^{-1}$ (Figura 1).

Pal et al. (1984), aplicando nitrato em concentração de $1 \%$, também não encontraram resultado satisfatório na indução floral em mangueira 'Dashehari'. Valente e Donadio (1993), visando à antecipação no florescimento de mangueira 'Tommy Atkins', no Estado de São Paulo, com aplicações de $\mathrm{KNO}_{3}$ nas concentrações se 2,3 e 4\%, também não encontraram diferenças significativas no florescimento. Já Barros et al. (1996), observando o efeito de $\mathrm{KNO}_{3}$ nas concentrações de 2, 4 e $6 \%$, constataram que o nitrato antecipou o florescimento em até 43 dias e intensificou significativamente a floração, embora não tenha havido diferenças significativas entre as concentrações e o número de aplicações do produto.

Neste trabalho não houve resposta significativa para a característica peso médio de frutos em nenhuma das plantas, em relação aos diferentes tratamentos. O peso medio geral ficou em torno de $0,445 \mathrm{Kg}$. Winston (1992), avaliando o efeito do PBZ no crescimento, florescimento e no rendimento de manga, cultivar Kensington Pride, também não encontrou resposta significativa em relação ao peso médio de frutos. Barros et al. (1996) também não observaram respostas significativas para o peso médio dos frutos em seu trabalho quando utilizaram três concentrações de $\mathrm{KNO}_{3}(2,4$ e $6 \%)$ com 1,2 e 3 aplicações na cultivar Tommy Atkins no sudoeste baiano.

Com relação ao número de frutos por planta, verificou-se que apenas o efeito dos tratamentos com paclobutrazol foram significativos. Na Figura 3, observa-se que o paclobutrazol apresentou boas respostas, aumentando significativamente o número de frutos por planta. No tratamento sem a aplicação desse inibidor, o número de frutos por planta ficou em torno de 56; já nos tratamentos com 1000 mg. $\mathrm{L}^{-1}$ e $1500 \mathrm{mg} . \mathrm{L}^{-1}$, houve um aumento para $213 \mathrm{e} 182$ frutos por planta, o que dá um percentual de 280 e $225 \%$ a mais no número de frutos por planta, respectivamente, em relação ao tratamento com dosagem de $0 \mathrm{mg} . \mathrm{L}^{-1}$.

Esse resultado vem confirmar a resposta do paclobutrazol no rendimento da planta, o que também foi confirmado por Winston (1992), Ferrari e Sergent (1996b) e Salazar-Garcia e Vazquez-Valdina (1997), que encontraram respostas positivas no aumento do número de frutos por planta ao analisarem trabalhos feitos em mangueira de diferentes cultivares, com aplicação do paclobutrazol.

Já os tratamentos com aplicações de $\mathrm{KNO}_{3}$ e ethephon não influenciaram nas resposta para o número de frutos por planta; resultados que assemelham aos de Valente e Donadio (1993), que verificaram a ineficiência do ethephon em promover o florescimento e rendimento da mangueira.

Observa-se mais uma vez que apenas as plantas que receberam o paclobutrazol apresentaram diferenças significativas para a característica produção de frutos por planta.

Esse aumento de produção com aplicação de paclobutrazol está diretamente ligado ao maior número de frutos por planta. Observando-se a Figura 4, verifica-se que, no tratamento sem a aplicação do paclobutrazol, a produção ficou em $23,9 \mathrm{~kg}$ de frutos por planta e, ao analisar os tratamentos com aplicação de $1000 \mathrm{mg} . \mathrm{L}^{-1} \mathrm{e}$ $1500 \mathrm{mg} . \mathrm{L}^{-1}$ de paclobutrazol, a produção em $\mathrm{kg} / \mathrm{planta}$ aumentou para 90,5 e 78,3, respectivamente. Houve um grande aumento da produção nas plantas que receberam dosagem de $1000 \mathrm{mg} . \mathrm{L}^{-1}$ em relação às plantas que receberam o paclobutrazol na dosagem de 1500 mg.L ${ }^{-1}$, ou seja, aproximadamente $12,2 \mathrm{~kg}$ a mais.

Também, apenas as plantas que receberam os tratamento com paclobutrazol apresentaram significância para a característica produção por hectare, já que essa característica está ligada diretamente com a produção de frutos por planta. No tratamento que não recebeu o paclobutrazol, a produção ficou em torno de 2,3908 t/ha. Já nos tratamentos com $1000 \mathrm{mg} . \mathrm{L}^{-1} \mathrm{e}$ $1500 \mathrm{mg} . \mathrm{L}^{-1}$, a produção ficou em torno de 9,0571 e $7,8316 \mathrm{t} / \mathrm{ha}$, tendo, assim, um ganho significativo em relação ao tratamento sem o paclobutrazol (Figura 4). Comparando-se a produção na dosagem de $1000 \mathrm{mg} . \mathrm{L}^{-1}$ com a produção na dosagem de $1500 \mathrm{mg} . \mathrm{L}^{-1}$ de paclobutrazol aplicado, observa-se que as plantas que receberam a dosagem de $1000 \mathrm{mg} . \mathrm{L}^{-1}$ tiveram um ganho superior em produção, em torno de 1,225 t/ha.

Essa menor produção alcançada no tratamento com $1500 \mathrm{mg} . \mathrm{L}^{-1}$ de paclobutrazol pode ter sido em função de uma superdosagem, o que pode ter acarretado algum efeito fisiológico na planta, prejudicando a sua produção, uma vez que essa dosagem é acima da recomendada pelo fabricante para na mangueira 'Tommy Atkins'. 


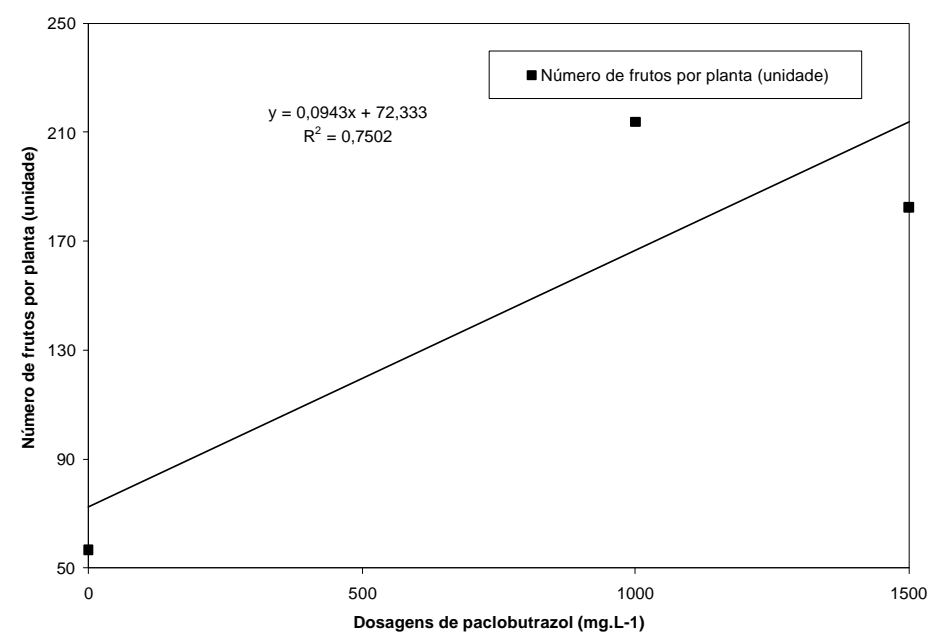

FIGURA 3 - Equação de regressão do tratamento com paclobutrazol para a característica número de frutos por planta. ESAM, Mossoró- RN. 2000.

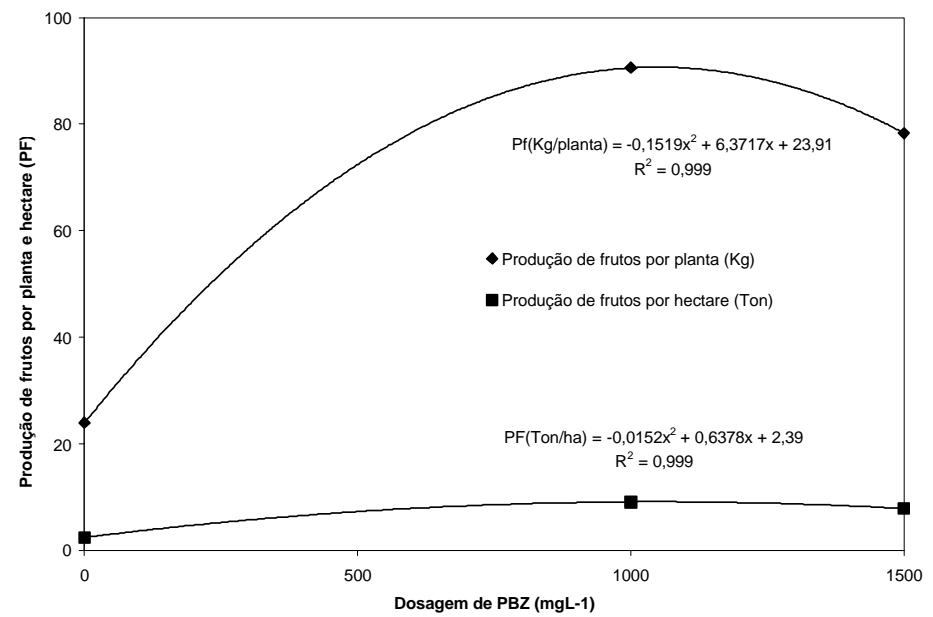

FIGURA 4 - Equação de regressão para o tratamento com PBZ para a característica produção de frutos por planta (Kg) e produção por hectare(ton). ESAM, Mossoró- RN. 2000.

\section{CONCLUSÕES}

a) O paclobutrazol foi eficaz na paralisação do crescimento vegetativo da mangueira;

b) A taxa de florescimento teve melhor resposta $(64,3 \%)$ na dosagem de $1000 \mathrm{mg} . \mathrm{L}^{-1}$ de paclobutrazol;

c) O número de frutos por planta, a produção e produtividade destacaram-se no tratamento com 1000 mg. $\mathrm{L}^{-1}$ de paclobutrazol;

d) $\mathrm{O}$ KNO3 e o ethephon, isoladamente, não influenciaram em nenhuma das características avaliadas.

\section{REFERÊNCIAS BIBLIOGRÁFICAS}

ALBERT, B.; LOSSOIS, P. Considérations sur la phénalogie des espècies frutières arbustives. Fruits, Paris, v. 27, p. 269-286, 1972.

ALBUQUERQUE, J. A. S.; MEDINA, V. A. D. Indução de floração em mangueira cv. Tommy Atkins com nitrato de amônio. In: CONGRESSO BRASILEIRO DE FRUTICULTURA, 11., 1991, Petrolina. Revista Brasileira de Fruticultura, Cruz das Almas, v. 13, p. 5-93, 1991. 
ALBUQUERQUE, L. A. S.; MOUCO, M. A.; REIS, V. C. Floração da mangueira através do uso de reguladores de crescimento. Petrolina: EMBRAPA, 1999. (Instruções Técnicas da Embrapa Semi-Árido, v. 12).

BARROS, P. G.; CUNHA, G. A. P.; REINHARDT, D. H.; FONSECA, N.; BARBOSA, N. M. L. Efeito do nitrato de potássio na floração e frutificação de mangueira (Mangifera indica L.) cv. Tommy Atkins no Sudeste da Bahia. Revista Brasileira de Fruticultura, Cruz das Almas, v. 14, p. 188-194, 1996.

CASTRO NETO, M. T. Informações técnicas sobre a cultura da manga no semi-árido brasileiro. [S.1.]: EMBRAPA, 1995. 173 p.

CUNHA, G. P.; SAMPAIO, J. M. M.; NASCIMENTO, A. S. do; SANTOS FILHO, H. P.; MEDINA, V. M. Manga para exportação: aspecto técnico da produção. Brasília: EMBRAPA-SPI, 1994. 35 p. (Série publicações técnicas FRUPEX, 8).

FERRARI, F. D.; SERGENT, E. A. Promocion de la floración y frutificacion del mango (Mangifera indica L.) cv. Haden, com Nitrato de Potasio. Revista da Facultad de Agronomia, Maracay, v. 22, p. 1-8, 1996.

FERRARI, F. D.; SERGENT, E. A. Promocion de la floración y frutificacion del mango (Mangifera indica L.) cv. Haden, com Paclobutrazol. Revista da Facultad de Agronomia, Maracay, v. 22, p. 9-17, 1996.

KURIAN, R. M.; IYER, C. P. A. Chemical regulation of tree size in mango (Mangifera indica L.) cv. Alphonso.I.Effects of growth retardant treatments on vegetative growth and tree vigour. Journal of Horticultural Science, Alexandria, v. 68, p. 349-354, 1993.

NORUSIS, M. J. SPSS statistics. IIIinois: SPSS, 1990.

NUÑEZ-ELIZEA, R.; CALDEIRA, M. L. Adelanto de la floración e cosecha em mango 'Haden' com aspersiones de nitrato de amônio. In: CONGRESSO BRASILEIRO DE FRUTICULTURA, 9., 1987, Campinas. Anais... Campinas: SBF, 1987. v. 2, p. 7-561.

PAL, R. N.; CHADKA, K. L.; RAO, M. R. K. Effect of different plant growth regulators and other chemicals on flowering behaviour of mango. Indian Journal Horticultural, New Delhi, v. 41, p. 8-15, 1984.
RABÊLO, J. E. de S. Florescimento e frutificação de mangueira (Mangifera indica L.) 'Haden' em resposta a anelamento e aplicação de ethephon e nitrato de potássio. 1995. 84 f. Dissertação (Mestrado em Agronomia) - Universidade Federal de Viçosa, Viçosa, 1995.

REIS, V. C. S. Efeito da aplicação foliar do paclobutrazol na floração e frutificação da mangueira (Mangifera indica L.) cv. Tommy Atkins. 1999. Dissertação (Mestrado em Agronomia) - Universidade Federal da Bahia, Salvador, 1999.

SALAZAR-GARCIA, S.; VAZQUEZ-VALDINA, V. Physiological persistence of paclobutrazol on the Tommy Atkins mango (Mangifera indica L.) under rainfed conditions. Journal of Horticultural Science, Alexandria, v. 72, n. 2, p. 339-345, 1997.

SANTANA, J. R. F.; CUNHA, G. A. P.; FONSECA, N.; SOUTO, R. F. Efeito de indutores florais sobre o florescimento, frutificação e rendimento das cvs. de manga Van Dyke, Haden e Tommy Atkins no Norte de Minas Gerais II: nitrato de potássio. Revista Brasileira de Fruticultura, Cruz das Almas, v. 14, n. 3, p. 289296, dez. 1997.

SILVA, D. A. M.; VIEIRA, V. J. S.; MELO, J. J. L.; ROSA JÚNIOR, C. R. M.; SILVA FILHO, A. V. Mangueira (Mangifera indica L.): cultivo sob condição irrigada. Recife: SEBRAE, 1994. 42 p. (Agricultura, 9).

SIMÃO, S. Manual de fruticultura. São Paulo: Ceres, $1971.530 \mathrm{p}$.

TANAJURA FILHO, J. G. Indução de florescimento em mangueira. In: SIMPÓSIO ESTADUAL SOBRE PRODUÇÃO DE MANGA, 1., 1990, Vitória da Conquista. Anais... Vitória da Conquista: UESB, 1992. p. 5-83.

VALENTE, J. P.; DONADIO, L. C. Mango flower induction by $\mathrm{KNO}_{3}$ and ethephon spraying. In: INTERNATIONAL SYMPOSIUM ON TROPICAL FRUITS, 1., 1993, Vitória. Abstracts... Vitória: EMCAPA, 1993. p. 51.

WAMPLE, R. L.; CULVER, E. B. The influence of paclobutrazol, a new growth regulator, on sunflowers. Journal of the American Society for Horticultural Science, Alexandria, v. 108, p. 122-125, 1983. 
WINSTON, E. C. Evaluation of paclobutrazol on growth, flowering and yield of mango cv. Kensington Pride. Australian Journal Exp. Agriculture, [S.1.], v. 32, p. 97-104, 1992.
WINSTON, E. C.; WRIGHT, R. M. Mango flower induction: ethephon, potassium nitrate and cincturing. In: AUSTRALIAN MANGO RESEARCH WORKSHOP, 1., 1984, Cairns. Proceedings... Cairns: CSIRO, 1984. p. 57-70. 